$\xi_{p}$

\title{
Dynamic Reliability Analysis of Corroded Pipeline Using Bayesian Network
}

\author{
Nurul Sa'aadah Sulaiman ${ }^{1 *}$, Henry Tan $^{2}$ \\ ${ }^{1}$ Faculty of Chemical \& Natural Resources Engineering, Universiti Malaysia Pahang \\ ${ }^{2}$ School of Engineering, University of Aberdeen, UK \\ *Corresponding authorE-mail: saaadah@ump.edu.my
}

\begin{abstract}
Maintenance and integrity management of hydrocarbons pipelines face the challenges from uncertainties in the data available. This paper demonstrates a way for pipeline remaining service life prediction that integrates structural reliability analysis, accumulated corrosion knowledge, and inspection data on a sound mathematical foundation. Pipeline defects depth grows with time according to an empirical corrosion power law, and this is checked for leakage and rupture probability. The pipeline operating pressure is checked with the degraded failure pressure given by ASME B31G code for rupture likelihood. As corrosion process evolves with time, Dynamic Bayesian Network (DBN) is employed to model the stochastic corrosion deterioration process. From the results obtained, the proposed DBN model for pipeline reliability is advanced compared with other traditional structural reliability method whereby the updating ability brings in more accurate prediction results of structural reliability. The comparisons show that the DBN model can achieve a realistic result similar to the conventional method, Monte Carlo Simulation with very minor discrepancy.
\end{abstract}

Keywords: Bayesian network; corrosion prediction; dynamic reliability; pipeline integrity; reliability analysis.

\section{Introduction}

Corrosion represents increasing challenges for the operation of subsea pipelines and become the major threats posed to offshore oil and gas pipelines in relation to operational integrity. In fact, this becomes even more prevalent as the pipeline increases in age. Bai \& Bai [1] defined corrosion as a deterioration of metal owing to chemical or electrochemical reactions between the metals and its surrounding. In this instance, the direct outcome of the corrosion activity would typically result in metal loss directly to the pipelines wall thickness, and as this progress with time, a corresponding reduction in the integrity, safety and the structural reliability of the pipeline to withstand the applied operational stresses. This corrosion problem can lead to leakage of the pipeline or even worse, rupture may occur.

Reliability analysis has become a vital tool for assessment and mitigation of threats due to corrosion deterioration in pipelines. The analysis is performed to evaluate how the integrity of a pipeline is affected by a corrosion defect and predicting the probability to fail as a result of corrosion defect growth. This probabilistic approach has been extensively used in the last decades within the pipeline industry as it is proven to provide a reasonable framework to account for various uncertainties that impact the development of suitable maintenance strategies [2]-[6]. It is as well important to model the corrosion growth in order to predict corrosion deterioration in engineering structure (e.g. bridges, pipelines, and nuclear power plant facilities) [5]-[9]. A more recent method of probabilistic approach is the causal probabilistic networks also known as Bayesian Networks (BN). BN can be used to determine system reliability by considering the associated uncertainties [10] and are computationally efficient in updating performance when new knowledge becomes available [9], [11]. Moreover, the extensions of BNs, known as Dynamic Bayesian Network (DBN) can be used to represent the discrete-time stochastic process. They are formed by a series of static BNs called time slices. DBN can be used to model stochastic deterioration model similar to that of a Markov model. The major advantages of $\mathrm{BN}$ and its extensions are that they have the capabilities of updating and considering multiple parameters at a time for a realistic deterioration model [9]. A number of researchers have applied $\mathrm{BN}$ for deterioration modelling [9; 11-13] however this paper focuses on the evaluation of a pipeline's reliability subjected to stochastic corrosion deterioration using DBN approach.

\section{Bayesian Network Theory}

A BN is formed by random variables together with directed arcs and is associated with joint probability distributions. This BN is used to represent uncertain knowledge in Artificial Intelligence [14]. In the model, each node represents a variable that can be in one of a finite state. Meanwhile, the arc linking two variables designates the causal or influential relationships between them. The relationship can be calculated by applying the chain rule for Bayesian networks. Given a set of random variable $X=X_{1}, X_{2}, \ldots, X_{n}$ within a $\mathrm{BN}$, a unique joint probability distribution of the entire network over all the variables is given by the product of conditional distributions attached to each node as:

$$
P(X)=P\left(X_{1}, \ldots, X_{n}\right)=\prod_{i=1}^{n} P\left[X_{i} \mid \text { parents }\left(X_{i}\right)\right]
$$

Bayesian network can be expanded into Dynamic Bayesian Net- 
work (DBN) to characterise system behaviour that evolves with time. Compared with BN, the DBN is more appropriate for observing and predicting values of random variables and allows the representation of the system state at any time [15]. A DBN represents the probability distribution over the states of random variable for the period of $[0, j]$. In this study, the DBN is expressed as a pair that comprises a Bayesian network model that defines the prior $P\left(X_{j}\right)$ and a two-slice temporal Bayes net that defines $P\left(X_{j} \mid X_{j-1}\right)$ by means of a transition probability table, defined by the following term [16]:

$$
P\left(X_{j} \mid X_{j-1}\right)=\prod_{i=1}^{N} P\left(X_{j}^{i} \mid p a\left(X_{j}^{i}\right)\right)
$$

Here, $X_{j}^{i}$ is the $i$ th node at time $j(i=1,2, \ldots, N)$, and $p a\left(X_{j}^{i}\right)$ denote the parents of $X_{j}^{i}$ in the Bayesian network. This model follows Markovian property in which the future slice $(j+1)$ is conditionally independent of the past $(j-1)$ given the present $(j)$. Each time slice is treated as a separate Bayesian networks and the joint probabilities distribution of the nodes within the $J$ time slices can be obtained by unrolling the network expressed as:

$$
P\left(X_{1: J}\right)=\prod_{j=1}^{J} \prod_{i=1}^{N} P\left(X_{j}^{i} \mid p a\left(X_{j}^{i}\right)\right)
$$

\section{Network Model Development}

The development of DBN model for pipeline structure reliability consists of two parts: structural reliability and growth of corrosion defect depth.

\subsection{Structural Reliability}

The objective of structural reliability is to evaluate the safety of the structure by determining whether the stress exerted to a structure exceeds its strength or not. Suppose a general pipeline of which structural reliability is dependent on a set of limit state functions $g=\left\{g_{1}, \ldots, g_{n}\right\}$. This limit states function $g$ is expressed by the difference between pipeline resistance $r$ and stress on pipeline $s$. This generic state function can represent different limit states defined according to different failure modes parameters. A corroding hydrocarbon pipeline can fail by leakage or rupture, depending on the size of the through-wall defect [5]. Thus these two failure modes were considered. The Bayesian networks model shown in Figure 1 was adopted to depict the overall pipeline structure with the two types of failure modes.

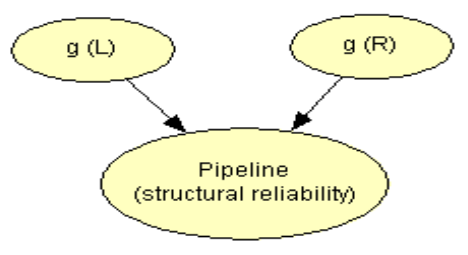

Fig. 1: Bayesian networks model for structural reliability of a general pipeline structure based on different limit state functions.

The limit state functions $g$ for leakage is expressed by the difference between the pipeline thickness and the corrosion defect depth. Leakage failure occurs when corrosion depth has reached the maximum allowable thickness ( $80 \%$ of the pipeline wall thickness) [5], [6]. Therefore, the limit state function for leakage pipeline $g(L)$ is given as:

$$
\begin{aligned}
g(L) & =r-s \\
& =0.8 t-d
\end{aligned}
$$

where $t$ is wall thickness of the pipeline and $d$ is the corrosion defect depth. It should be bear in mind that the if the pipeline suffered with the defects depth of more than $80 \%$ of the pipeline wall thickness, this does not necessarily mean that leaks will occur for an isolated defect. However, a defect of this extent is considered a serious integrity issue and is not tolerated under any circumstances in the field of petroleum industry [5].

On the other hand, the rupture mode is defined as the difference between the pipeline failure pressure $p_{F}$ and the pipeline internal operating pressure $p_{o} . p_{F}$ is generally determined by failure pressure model. The basic equation of failure pressure model depends on the pipe outer diameter $D$, the pipe wall thickness $t$ and the hoop stress at failure $S$. The limit state function for rupture $g(R)$ is written as:

$$
\begin{aligned}
g(R) & =r-s \\
& =p_{F}-p_{o}
\end{aligned}
$$

In this study, modified ASME B31G code [17] was utilised. For a pressurized pipeline where pipe wall thickness is relatively small compared to pipe outer diameter and the fluid density is relatively low compared to the fluid pressure [18], the B31G code gives the failure pressure $p_{F}$ as:

$p_{F}=2 \frac{S t}{D}$

$$
\begin{aligned}
& \text { Where } \\
& S=(Y S+68.95) \frac{1-\left(A / A_{0}\right)}{1-\left(A / A_{0}\right) M^{-1}} \\
& A / A_{0}=0.85 \frac{d}{t} \\
& M= \begin{cases}\sqrt{1+0.63 \frac{l^{2}}{D t}-0.0034 \frac{l^{4}}{D^{2} t^{2}}} & \frac{l^{2}}{D t} \leq 50 \\
0.032 \frac{l^{2}}{D t}+3.3 & \frac{l^{2}}{D t}>50\end{cases}
\end{aligned}
$$

where $Y S$ is specific minimum yield strength of the material (in the unit of MPa), $A$ is the area of the defect, $A_{0}$ is the original cross-sectional area of the pipe at the defect, $l$ is the length of corrosion, $d$ is corrosion defect depth and $M$ is the Folias factor or also known as bulging factor. The value of $A / A_{0}$ is taken as the average of parabolic and rectangular geometry of corrosion depth.

\subsection{Growth of Corrosion Defect Depth}

An internal corrosion defect is expected to grow with increasing exposure period if no countermeasure is taken [19]. In order to estimate the future magnitudes of corrosion defects, it is essential to assess the probabilistic distribution of corrosion defect growth rate [20]. In addition, prediction requires a failure model having the collected and forecast corrosion data input, to determine the pipeline's probability of failure within a given timeline [5].

There are various corrosion defect-growth models had been used to describe the loss of wall thickness with time of exposure [21] [26]. However, a widely acceptance and practical engineering approach to account for corrosion defect propagation in a pipeline is to use a power function. The time evolution of corrosion defect depth, $d$ after $T$ years is described by the corrosion power law written as:

$d=k T^{q}$ 
Where $d$ is the depth of the corroded layer in mm, $T$ is the exposure time (year) and $k$ and $q$ are corrosion constants. The $k$ and $q$ values are to be calculated by fitting equation (7) from field data [7]. From the corrosion growth equation, the defect depth can be calculated for structural reliability estimation in the pipeline.

As deterioration is a stochastic process, it is important to model the time variation in pipeline reliability. In this research, DBN was employed to model stochastic corrosion deterioration process as a discrete time process. Equation (7) was employed as the basis for DBN modelling of the pipeline internal corrosion deterioration process. When modelling a system that evolves with time, random variable at a given time instant or so called as a time slice typically depends on the state of the system at past time instants. By definition in this presented work, the present elapsed time $T$ is conditional on previous elapsed time $T_{\text {previous }}$. In Hugin Expert software, the dynamic Bayesian networks are represented by temporal clone $T_{\text {previous }}$ in order to specify time dependencies for the regular nodes. Both of these time nodes are discrete time variables. Figure 2 presents the equivalent DBN model where $d$ is the corrosion defect depth after $T$ years, and $k$ and $q$ are the corrosion constants. The number of time slices denoted the number of exposure years for the pipeline. Using the concept of DBN, the corrosion defect depth can be projected till year $T_{n}$.

In order to improve the corrosion deterioration prediction, additional available parameters regarding maintenance actions were considered for information updating. The model updating consists of creating a maintenance variable node as in Figure 2.

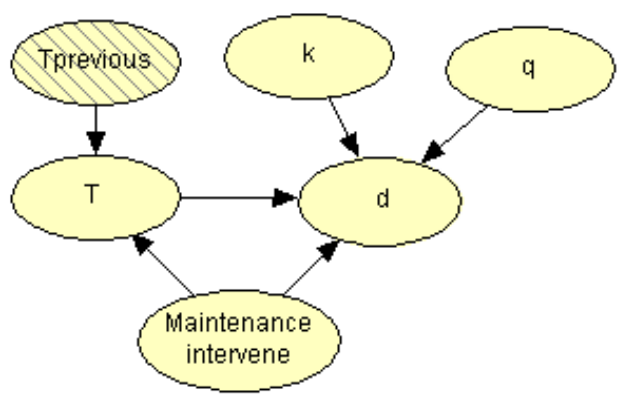

Fig. 2: DBN model of corrosion growth with integration of maintenance variable.

A maintenance intervenes, on the other hand, was introduced and carried out to restore or maintain the pipeline to the tolerable operational condition. Maintenance has an impact on the deterioration process that then affects the reliability of the pipeline. The outcome quantification on pipeline deterioration from the CPT can be achieved in two ways. In the beginning, the maintenance was considered as a revision of the probability density associated with the states of the corroded pipeline. Then, the repair acts on the deterioration process in which parameters were updated each time maintenance intervene on the pipeline occurs [27]. These revisions or modifications are directly related to the effectiveness of the maintenance action which three types of actions are typically renowned.

- $\quad$ No or minimal maintenance (as bad as old): this maintenance action set the pipeline in the same state as it was before. The degradation process is unchanged and the state of the pipeline before and after the intervention is similar.

- Perfect maintenance (as good as new): after each maintenance intervenes, the pipeline that goes through perfect maintenance is considered new and no corrosion present. In the model, the pipeline is restored to its nominal state at that particular intervention time and the time variable $T$ is reset to zero.

- Imperfect maintenance: this maintenance action is generally in between as good as new and as bad as old. In this study, imperfect maintenance reduces the age (time variable $T$ ) of the pipeline, better than it was, however, worse than it could be as new.

By now, the modelling of structural reliability and corrosion defect growth were modelled. These two parts were then combined to implement time-variant structural reliability of pipeline structure. Figure 3 illustrates the DBN model of pipeline structural reliability

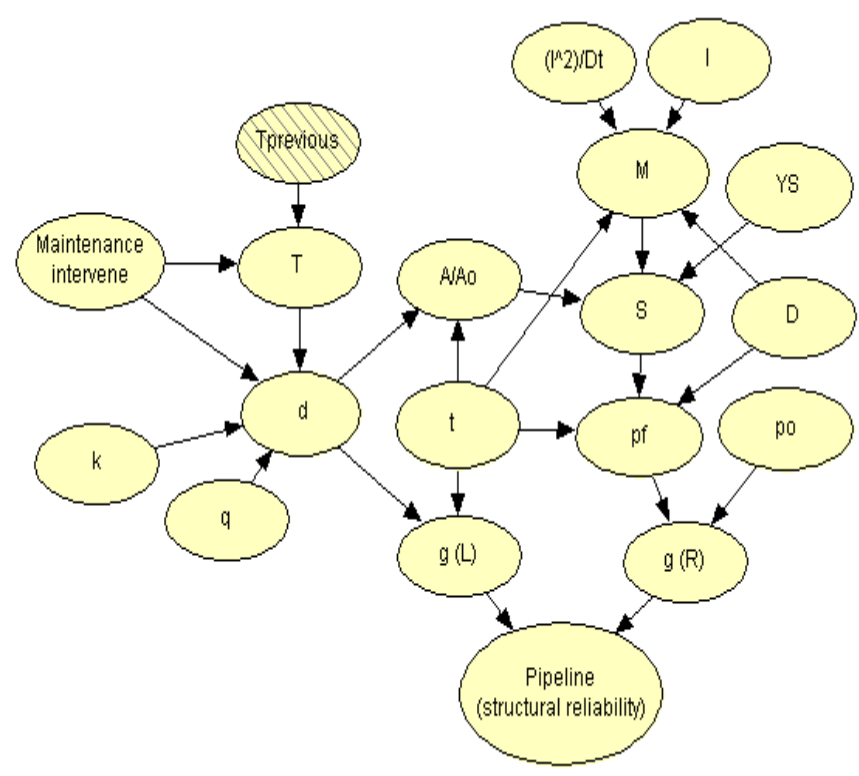

Fig. 3: Structural reliability of pipeline as a DBN

\section{Parameter Estimation}

Parameters estimation in this study was mainly constructed based on the existing literature and deterministic equations. The prior probabilities were attained from the existing literature directly. The CPTs on the other hand were formulated from the deterministic equations since the modelling of structural reliability was developed based on limit state functions. In fact, the relationship expressed by the deterministic equations was encoded directly into CPTs using Hugin Expert software. This is done by utilising the inbuilt arithmetic operations and statistical distributions in Hugin Expert that can considerably help in creating the CPTs. The Equation (4) describing the corrosion defect growth in the pipeline was as well applied to estimate the CPTs related the deterioration model.

\section{Case Study}

The proposed DBN model was demonstrated to a hydrocarbon pipeline studied by Ahammed [4]. Some of the information of the previous study were directly utilised for the application in this presented work. The DBN model was employed to predict the structural reliability of the pipeline for an exposure period of 50 years. It must be bear in mind that the purpose of this analysis was to illustrate the methodology. In this demonstration, the transparency and ability of the network to determine the reliability of the pipeline were examined.

The reliability analysis of this case study was based on the Dynamic Bayesian network of structural pipeline reliability analysis established in Figure 3. The established DBN model was compared to a conventional structural reliability method, for instance, MCS. Furthermore, the accuracy and advantages of proposed DBN model as a robust model over the conventional method were illustrated in this application. 


\subsection{Parameters Estimation for DBN Model}

The limit state functions employed in this study are functions of many basic parameters and were considered to be random and statistically distributed. For this methodology, all the parameters were characterized only by their means, $\mu$ and coefficient of variation (C.O.V). The numerical values and the distributions types of the parameters of this example pipeline were based on the realistic available relevant information from a reference [4]. The value for the multiplier $k$ was assumed to be 0.318 meanwhile, the mean and standard deviation for the power $n$ are 0.6 and 0.17 , respectively. The $k$ value was empirically obtained from the last inspection data for the pipeline that was carried out in the year 10 and the corresponding values of $d$ and $l$ were measured at that time [4]. The basic parameters listed in Table 1 have been utilized to investigate the reliability of the corroded pipeline. The modelling design in this work excluded evolution of the corrosion defect length Changes in the defect length have little or no influence on the estimation of the failure probability associated with individual corrosion defects [5]. Hence, the defect length in the example was treated as deterministic. In addition, this assumption will ease the analysis and will be less time consuming.

In this example, most of the basic parameters are continuous random variables and cannot be used directly. This is because most of the machine learning algorithms especially commercially available software are not designed to handle numeric attributes [28]. The available Bayesian networks software commonly requires the variable to be discrete in such a way to facilitate the inference algorithm for CPTs estimation as it can approximate the continuous state in a simple and convenient manner. The process of transforming numerical continuous variables into discrete variables requires the variables' data to be partitioned. The objective of discretization, in general, is to substitute the continuous variable with a finite number of discrete states while maintaining the most important properties of the function.

Discretization of equal length interval approach is used in this application with each variable having a different number of intervals. The discretization scheme for the parameters in Table 1 is obtained and summarised in Table 2. The CPTs associated with the root nodes of the DBN were computed based on the prior probability density functions of the corresponding random variables. By means of the discretized variables, the new CPTs can be determined based on the deterministic equations. This discretization process was supported by the Hugin Expert software which in fact runs the sampling algorithms for discretization.

Table 1: Probability distribution of input parameters used in Hugin Expert and Monte Carlo Simulation models [4].

\begin{tabular}{|c|c|c|c|c|}
\hline Parameter & \multicolumn{4}{|c|}{ Distribution characteristics } \\
\hline Symbol & Description & Mean & C.O.V & Type \\
\hline$Y S$ & $\begin{array}{c}\text { Material yield } \\
\text { strength }\end{array}$ & $\begin{array}{c}423 \\
\mathrm{MPa}\end{array}$ & 0.1 & Normal \\
\hline$t$ & Pipe wall thickness & $\begin{array}{c}10.0 \\
\mathrm{~mm}\end{array}$ & 0.05 & Normal \\
\hline$p_{o}$ & Operating pressure & $8 \mathrm{MPa}$ & 0.10 & Normal \\
\hline$q$ & $q$ & 0.6 & 0.17 & Normal \\
\hline$k$ & $k$ & 0.318 & - & Deterministic \\
\hline$l$ & Defect length & 200 & - & Deterministic \\
& $\mathrm{mm}$ & & Deterministic \\
\hline$D$ & $\begin{array}{c}\text { Pipe external diame- } \\
\text { ter }\end{array}$ & 600 & - & \\
\hline
\end{tabular}

Table 2: Discretization scheme of basic parameters.

\begin{tabular}{|c|c|c|c|}
\hline Variable & Probable range & $\begin{array}{c}\text { Number of } \\
\text { intervals }\end{array}$ & $\begin{array}{c}\text { Final interval } \\
\text { boundaries }\end{array}$ \\
\hline$T$ & $0-60$ & 60 & $0: 1: 60$ \\
\hline$Y S(\mathrm{MPa})$ & $300-550$ & 27 & $0,300: 10: 550, \infty$ \\
\hline$t(\mathrm{~mm})$ & $8.8-11.2$ & 42 & $0,8.8: 0.06: 11.2, \infty$ \\
\hline$p_{o}(\mathrm{MPa})$ & $5-11$ & 14 & $0,5: 0.5: 11, \infty$ \\
\hline$q$ & $0-1$ & 21 & $0: 0.05: 1, \infty$ \\
\hline
\end{tabular}

The safety measures of the pipeline reliability were determined based on limit state function $g(L)$ and $g(R)$ using equation (4) and (5), respectively. To simplify the model, the values generated from the safety measure calculations were summarised within the software in terms of probability distribution whether the pipeline is safe $(g>0)$ or fail $(g<0)$. Then, the CPT in Table 3 was used to represent the relationship of the overall pipeline structural reliability. The CPT values, however, can be changed by setting different probabilities between 0 and 1 in order to consider the real case. Thus, different types of probabilities of failure relationships can be modelled appropriately. In this application, the pipeline reliability was estimated for another 50 years from the last pipeline inspection. The inference algorithm for the structural reliability was executed with the support from Hugin Expert software.

Table 3: CPT model for pipeline failure based on the logic $(L \cup R)$
\begin{tabular}{|l|c|c|c|c|c|}
\hline & $g(L)$ & \multicolumn{2}{|c|}{$>0$} & \multicolumn{2}{|c|}{$\leq 0$} \\
\hline & $g(R)$ & $>0$ & $\leq 0$ & $>0$ & $\leq 0$ \\
\hline Pipeline & Safe & 1 & 0 & 0 & 0 \\
\cline { 2 - 6 } & Fail & 0 & 1 & 1 & 1 \\
\hline
\end{tabular}

\subsection{Result of DBN Model}

The aim of this section was to compare the performance of DBN model with existing structural reliability methods such as Monte Carlo Simulation (MCS). For MCS, the sampling numbers are 100 000 for each continuous variable. When quantified in Hugin Expert, the proposed DBN model was compiled in a few seconds. Furthermore, the output was the marginal probability of all variables, allowing the user to follow the distribution of failure probability through the time slices.

It should be emphasized that the limit state functions, $g(L)$ and $g(R)$ were all time-dependent due to the fact that corrosion defect depth grows with time and result in a reduction of the pipeline wall thickness and hence the resistance to operational pressure. The outcomes obtained from the DBN were compared to outcomes found from MCS, in term of reliability index $\beta$.

From the outcomes shown in Figure 4, it was observed that both the outcomes from DBN model and MCS signify the similar trends of reliability indexes. This is as expected that the reliability index $\beta$ decreases with exposure time, $T$ and correspondingly, the failure probability increases with time. It is explained in such a way that as the service year increases, the area of corrosion defect $A / A_{o}$ increases. Thus, the pipeline capacity to resist the effect of exerted stress generated by external loads is as well reduced. As can be seen from the plot, the two results show minor discrepancies but keeping close to one another at all the time. Since the result calculated from MCS are an approximate prediction of structural reliability rather than accurate assessment, the discrepancies never hinder the estimation result from DBN model to be a sensible evaluation. the comparison shows that the DBN model can achieve a reasonable result similar to the conventional method, MCS.

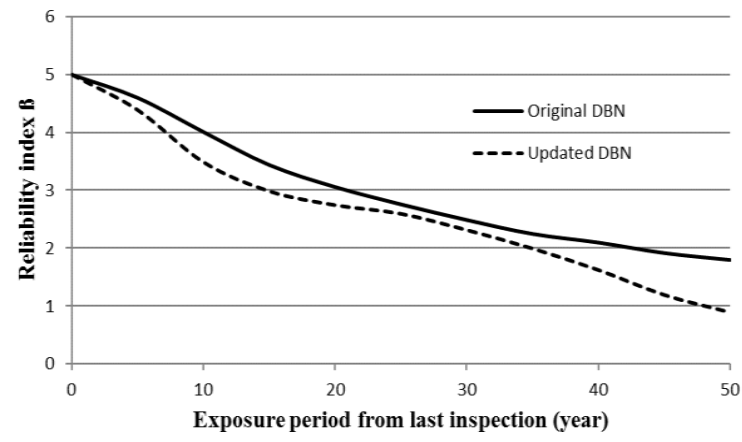

Fig. 4: Predicted reliability of pipeline failure for an exposure period of 50 years. 
Another updating example is by introducing a maintenance variable into the proposed model. The maintenance schemes tabulated in Table 4 which are no maintenance, imperfect maintenance, and perfect maintenance were simulated. The perfect maintenance is presumed to remove all corrosion loss and renew the pipeline. Imperfect maintenance, on the other hand, is presumed to have $50 \%$ opportunity to compensate corrosion loss and to repair the pipeline.

Table 4: Maintenance schemes on pipeline during 50 years.

\begin{tabular}{|c|c|c|c|}
\hline Time, $T$ (years) & 15 & 25 & 45 \\
\hline $\begin{array}{c}\text { Maintenance } \\
\text { scheme }\end{array}$ & $\begin{array}{c}\text { Imperfect } \\
\text { maintenance }\end{array}$ & $\begin{array}{c}\text { Perfect mainte- } \\
\text { nance }\end{array}$ & $\begin{array}{c}\text { Perfect } \\
\text { maintenance }\end{array}$ \\
\hline
\end{tabular}

Maintenance of pipeline structure can be in many forms such as corrective or preventive maintenance. The selection of maintenance activity on the pipeline is dependent upon the actual condition of the defects that occur. In this section, a very general corrective maintenance action is used as a case study to demonstrate the ability to update the proposed model. Figure 5 shows the updated pipeline reliability when the three categories of corrective maintenance schemes are implemented. It could be seen that at the year of 15 where imperfect maintenance took place, the reliability index is slightly increased. Whereas when the maintenance is perfect, the reliability index is shifted to the original value showing that there is no corrosion. Through the updating process, the outcome in Figure 5 can be applied for life extension purpose. The updated reliability shows that the life of pipeline can be extended through integrity maintenance. The operator could have an idea regarding the life that could be extended when a certain maintenance intervenes is applied. It is illustrated from the outcomes that the updating ability brings in more accurate prediction results of structural reliability, which eventually benefits pipeline maintenance optimization.

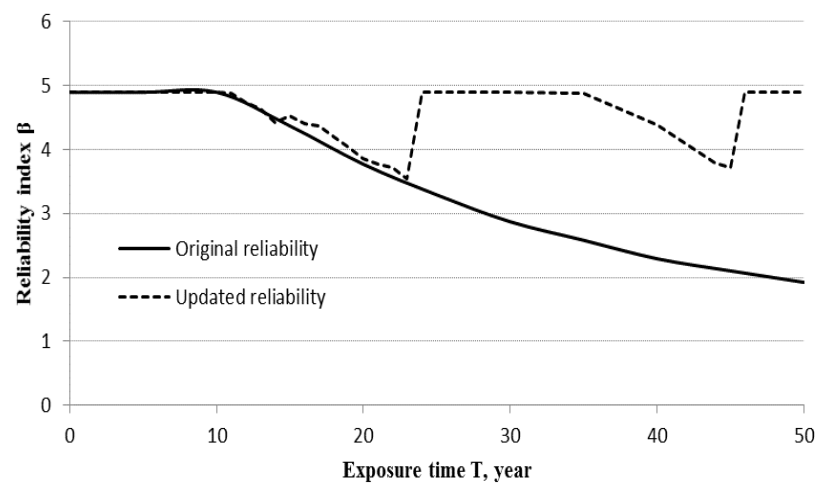

Fig. 5: Updated reliability index based on simulated maintenance schemes.

Based on the results obtained, the proposed DBN model for pipeline reliability is advanced compared with other traditional structural reliability method. On the other hand, the comparisons show that the DBN model can achieve a reasonable result similar to the conventional method, MCS. It was also demonstrated that the updating of the probability distribution of model parameters based on new information is straightforward with the DBN.

\section{Conclusion}

This paper presents the development of a Dynamic Bayesian Networks model to evaluate the time-dependent structural reliability of hydrocarbon pipeline subject to corrosion. The model was established through two parts: structural reliability and Dynamic Bayesian networks. Two types of corroded pipeline failure modes were considered for limit state functions namely leak and rupture. Dynamic Bayesian network, on the other hand, was employed to model the discrete-time stochastic process of corrosion depth growth. In order to verify the proposed model, an application was given based on previous study.

It was found that the advantages of this approach are significant, thus demonstrating the potential of the modelling technique. It is shown that dynamic Bayesian networks are able to model the important aspects of structural reliability methods as compared to Monte Carlo Simulation outcomes. The properties of the dynamic Bayesian network were exploited to obtain advantages with respect to insertion of evidence or new knowledge and updating of the dynamic model. From the results, it shows that the model improves the reliability updating in light of maintenance intervene. The inclusion of the evidence may help to reduce the prediction uncertainty. It is suggested that the resulting information can be used to set up an effective and economic inspection/maintenance plan. In fact, the model is useful in dealing with life extension with limited inspection data available, which is a challenging issue for pipeline integrity management nowadays especially for offshore lines.

\section{Acknowledgement}

The authors would like to express appreciation for the support of the sponsor Universiti Malaysia Pahang Internal Grant [RDU1703169].

\section{References}

[1] Bai Y \& Bai Q. (2014). Subsea Pipeline Integrity and Risk Management. Subsea Pipeline Integrity and Risk Management. Elsevier.

[2] Ahammed M \& Melchers RE. (1996). Reliability estimation of pressurised pipelines subject to localised corrosion defects. International Journal of Pressure Vessels and Piping, 69(3), 267272.

[3] Pandey MD. (1998). Probabilistic models for condition assessment of oil and gas pipelines. NDT and E International, 31(5), 349-358.

[4] Ahammed M. (1998). Probabilistic estimation of remaining life of a pipeline in the presence of active corrosion defects. International Journal of Pressure Vessels and Piping, 75(4), 321-329.

[5] Caleyo F, González JL \& Hallen JM. (2002). A study on the reliability assessment methodology for pipelines with active corrosion defects. International Journal of Pressure Vessels and Piping, 79(1), 77-86.

[6] Zhang S \& Zhou W. (2013). System reliability of corroding pipelines considering stochastic process-based models for defect growth and internal pressure. International Journal of Pressure Vessels and Piping, 111-112, 120-130.

[7] Amirat A, Mohamed-Chateauneuf A \& Chaoui K. (2006). Reliability assessment of underground pipelines under the combined effect of active corrosion and residual stress. International Journal of Pressure Vessels and Piping, 83(2), 107117.

[8] Valor A, Caleyo F, Hallen JM \& Velázquez JC. (2013). Reliability assessment of buried pipelines based on different corrosion rate models. Corrosion Science, 66, 78-87.

[9] Straub D. (2009). Stochastic modeling of deterioration processes through dynamic bayesian networks. Journal of Engineering Mechanics, 135(10), 1089-1099.

[10] Langseth H \& Portinale L. (2007). Bayesian networks in reliability. Reliability Engineering and System Safety, 92(1), 92-108.

[11] Rafiq MI., Chryssanthopoulos MK \& Sathananthan S. (2015). Bridge condition modelling and prediction using dynamic Bayesian belief networks. Structure and Infrastructure Engineering, 11(1), 38-50.

[12] Friis-Hansen A. (2000). Bayesian Network as a Decision Support Tool in Marine Applications. Technical University of Denmark.

[13] Wang R. (2012). Integrated health prediction of bridge systems using dynamic object oriented bayesian networks. Lcp-Bms. Queensland University of Technology.

[14] Jensen FV. (2001). Bayesian networks and decision graphs. Bayesian Networks and Decision Graphs.

[15] Wu WS, Yang CF, Chang JC, Château PA \& Chang YC. (2015). Risk assessment by integrating interpretive structural modeling and Bayesian network, case of offshore pipeline project. Reliability 
Engineering \& System Safety, 142, 515-524.

[16] Sahraoui Y, Khelif R \& Chateauneuf A. (2013). Maintenance planning under imperfect inspections of corroded pipelines. International Journal of Pressure Vessels and Piping, 104, 76-82.

[17] PARLOC. (2001). PARLOC 2001: The Update of Loss of Containment Data for Offshore Pipelines.

[18] Melchers RE \& Ahammed M. (2006). Statistical characterization of corroded steel plate surfaces. Advances in Structural Engineering, 9(1), 83-90.

[19] Mustaffa Z. (2011). System Reliability Assessment of Offshore Pipelines. Delft University of Technology.

[20] Caleyo F, Valor A, Venegas V, Hernandez JHE, Velazquez JC \& Hallen JM. (2012). Accurate corrosion modeling improves reliability estimations. Oil and Gas Journal, 110(10), 122-129.

[21] Alamilla JL \& Sosa E. (2008). Stochastic modelling of corrosion damage propagation in active sites from field inspection data. Corrosion Science, 50(7), 1811-1819.

[22] Velázquez JC, Caleyo F, Valor A \& Hallen JM. (2009). Predictive model for pitting corrosion in buried oil and gas pipelines. Corrosion, 65(5), 332-342.

[23] Hairil Mohd M \& Paik JK. (2013). Investigation of the corrosion progress characteristics of offshore subsea oil well tubes. Corrosion Science, 67(0), 130-141.

[24] Qian G, Niffenegger M \& Li S. (2011). Probabilistic analysis of pipelines with corrosion defects by using FITNET FFS procedure. Corrosion Science, 53(3), 855-861.

[25] Song FM. (2009). Predicting the mechanisms and crack growth rates of pipelines undergoing stress corrosion cracking at high $\mathrm{pH}$. Corrosion Science, 51(11), 2657-2674.

[26] K. Oliver and G. John, "Pipeline inspection - Conclusion: Corrosivity modeling helps determine current condition," Oil Gas J., vol. 105, no. 43, pp. 62-64, 2007. Oliver, K., \& John, G. (2007). Pipeline inspection - Conclusion: Corrosivity modeling helps determine current condition. Oil and Gas Journal, 105(43), 62-64.

[27] Muller A, Suhner MC \& Iung B. (2008). Formalisation of a new prognosis model for supporting proactive maintenance implementation on industrial system. Reliability Engineering and System Safety, 93(2), 234-253.

[28] Dawotola AW, Van Gelder PHAJM, Charima JJ \& Vrijling JK. (2011). Estimation of failure rates of crude product pipelines. In Applications of Statistics and Probability in Civil Engineering Proceedings of the 11th International Conference on Applications of Statistics and Probability in Civil Engineering (pp. 1741-1747). 